\title{
DER GUTE TON, ODER: \\ Die Funktion DANiEl KÜBlbÖCKS IM STAR-SySTEM VON DEUTSCHLAND SUCHT DEN SUPERSTAR UND IM ÖFFENTLICHEN DISKURS
}

\author{
Iris Stavenhagen
}

\begin{abstract}
Vorbemerkungen
Die Casting-Show Deutschland sucht den Superstar (DSDS) wurde nicht allein von der überwiegend jugendlichen Zielgruppe rezipiert, sondern fand auch Eingang in den öffentlichen Diskurs. Insbesondere auf den Drittplatzierten der ersten Staffel, Daniel Küblböck, wurde auffällig oft in verschiedenen Kontexten Bezug genommen. So findet er Erwähnung in einer Rede der thüringischen Wissenschaftsministerin Dagmar Schipanski zur Diskussion um Elite-Unis, um das Wettbewerbsprinzip exemplarisch ad absurdum zu führen:

»Wenn Frau Bulmahn glaubt, mit einem solchen Wettbewerb die von ihr propagierten Elite-Universitäten finden zu können, dann wird sie eine ähnliche Überraschung erleben wie der Gesangswettbewerb >Deutschland sucht den Superstarく mit Daniel Küblböck« (zit. n. N.N. 2004a).

In einem Artikel über Musikhochschulen dient Küblböck zur Veranschaulichung der Auffassung, dass seriöses Berufsmusikertum und Medienrummel nichts miteinander zu tun haben: »Daniel Küblböck hätte es hier nicht einmal bis zur Tür geschafft « (Schreiber 2003). Für den Musikpädagogen HansGünther Bastian steht er offenbar für die Korrumpierung musikalischer Wahrnehmung:

»Wir müssen das musikalische Analphabetentum unter Kindern und Jugendlichen stoppen, sie mit Kompetenzen ausstatten und damit immun machen gegen eine grassierende Küblböckitis in unseren mediokren Medien, die ihnen Hornhaut auf die ästhetische Seele brennen« (Bastian 2004).
\end{abstract}


Und der Psychologe Christian Lüdke fürchtet schädlichen Einfluss auf die jugendlichen Rezipienten: »Bei Daniel Küblböck ist es zu einem Entwicklungsrückstand gekommen. Das macht Gefahren für ihn, aber auch für seine Fans deutlich« (zit. n. N.N. 2004b).

Im Folgenden soll die Frage geklärt werden, wodurch Daniel Küblböck zum Stein des Anstoßes wurde und welche Rolle er im Gesamtgefüge von DSDS spielte. Bereits der Titel der Sendung suggeriert eine hohe Erwartungshaltung: »Deutschland « (also ein ganzes Land) »sucht« (hat offenbar einen dringenden Bedarf) »den Superstar (etwas Herausragendes, das sich von allen anderen - womöglich auch von Stars anderer Länder - abhebt). Der Umstrittenheitsgrad Küblböcks hatte wohl auch etwas damit zu tun, ob und inwieweit es diesem Kandidaten gelang, solche Erwartungen zu erfüllen, oder ob er - womöglich beabsichtigt - im Widerspruch zum Setting und den Normen dieses Casting-Formats stand.

Auf der Suche nach einer wissenschaftlichen Rezeption des Themas lässt sich feststellen, dass es bisher nur eine empirische Untersuchung hierzu aus dem Bereich der Medienwissenschaft gibt (Wolf 2004), die sich jedoch auf die Analyse des Markterfolges von DSDS als Modell für erfolgreiche Fernsehformate konzentriert und nicht die Absicht hat, einen Bezug zum gesellschaftlichen Kontext herzustellen. Das Phänomen der Stars fand im Bereich der Gesellschaftswissenschaften bisher geringe Beachtung. ${ }^{1}$ Im Kapitel »Kulturindustrie « der Dialektik der Aufklärung (Horkheimer/Adorno 1987: 144196), in den 1940er Jahren verfasst, finden sich jedoch bereits Elemente einer Analyse der Rolle von Stars, die auf deren Verwertung im Kontext einer kapitalistischen Wirtschaftsproduktion fokussiert. In den 1950er Jahren machte sich der französische Soziologe und Anthropologe Edgar Morin daran, die Funktions- und Wirkungsweisen des Star-Systems am Beispiel der Filmindustrie erstmals in einer systematischen Theorie zu erfassen. Aus seiner Beschreibung des Phänomens ergeben sich überraschende Parallelen zum Star-System der Casting-Shows der 1990er Jahre, auf die sich eine Analyse dieses vermeintlich jungen Phänomens aufbauen lässt. Eine gute Ergänzung hierzu bietet Schweppenhäuser (2004), der in seiner rezeptionstheoretischen Analyse medialer Ereignisse Ansätze der Kritischen Theorie, der Systemtheorie Luhmanns, der Cultural Studies und verschiedener Medientheoretiker des 20. Jahrhunderts vereint.

Die erste Staffel von DSDS habe ich von dem Zeitpunkt an verfolgt, als die Auswahl der letzten 30 Kandidaten begann. Als dokumentarische Quellen zu Küblböck und der Casting-Show habe ich unter anderem die Video-

1 Eine ausführliche Erörterung der Quellenlage zu Fan- und Startheorien findet sich in Wiediger 1996: 1-70. 
aufnahme eines Superstar-Specials vom Juni $2004^{2}$ herangezogen, das Auftritte aus der ersten Staffel, TV-Reportagen, Interviews und auch die weitere Karriere Küblböcks zusammenfasst und kommentiert, sowie das als Küblböck-Autobiografie vermarktete, von Julia Boenisch verfasste Buch Ich lebe meine Töne (2003). Daneben existiert von Ariane Aarberger (2003), einer Werbefachfrau, ein Buch über Küblböck, das aus der Perspektive eines weiblichen Fans jenseits des Teenager-Alters geschrieben wurde.

\section{Retrospektive: Das Star-System des Films}

Im Kulturindustrie-Kapitel der Dialektik der Aufklärung (Horkheimer/Adorno 1987: 144ff.) dienen Filmindustrie und Rundfunk in erster Linie als Hintergrund einer umfassenden Kritik an der kapitalistischen Produktionsweise von Kultur, die dem autonomen Subjekt keine Chance lässt: weder den Rezipienten noch den Darstellern. Selbst die organisierte Talentsuche in diesem Bereich diene letztlich ebenfalls der Aneignung des Publikums:

»Jede Spur von Spontaneität des Publikums im Rahmen des offiziellen Rundfunks aber wird von Talentjägern, Wettbewerben vorm Mikrophon, protegierten Veranstaltungen aller Art in fachmännischer Auswahl gesteuert und absorbiert. Die Talente gehören dem Betrieb, längst ehe er sie präsentiert: sonst würden sie nicht so eifrig sich einfügen« (ebd.: 146).

In der monopolisierten Kulturindustrie gibt es demnach keine wirklichen Differenzen; jede Art von Abweichung ist ebenfalls Bestandteil des Systems:

»Alle Verstöße gegen die Usancen des Metiers, die Orson Welles begeht, werden ihm verziehen, weil sie als berechnete Unarten die Geltung des Systems um so eifriger bekräftigen« (ebd.: 153).

Die Analyse des Star-Systems, wie Edgar Morin (1972) sie erarbeitet hat, beinhaltet keine explizite Kritik an der kapitalistischen Produktionsweise von Kultur. Er legt die These zu Grunde, dass das Phänomen der Stars einem anthropologischen Bedürfnis nach Mythen und Verehrung entspringt - ein irrationales Element in einer vermeintlich rationalen Gesellschaft, die vorgibt, Religion bzw. Mystik längst ad acta gelegt zu haben, und für die Kulte lediglich ein Element vermeintlich primitiver Gesellschaften sind. Morin plädiert dafür, diese Irrationalität nicht zu verwerfen und ihr auch in der seriösen Wissenschaft Aufmerksamkeit zu widmen.

2 Daniel Küblböck - Das Superstar-Special, SuperRTL, Sendetermin 6. Juni 2004, 150 Minuten Länge. 
Der Terminus »Star-System« bezeichnet das systematische Suchen und Aufbauen von Stars (inklusive der vollständigen Verfügung über deren Privatleben und der Verbreitung entsprechender Marketing-Artikel). Die konkrete Gestalt des Star-Systems, wie sie sich zuerst im Hollywood der 1940er Jahre gezeigt habe, sei ein Produkt des Kapitalismus: Stars waren nicht nur Prestige-, sondern auch Kapitalgewinn für die produzierenden Filmgesellschaften und wurden in Zeiten zunehmender Monopolisierung dieser Gesellschaften als Verkaufsargument dringend benötigt. Die Möglichkeit industrieller Reproduktion verhalf zur massenhaften Verbreitung eines Star-Images und aller Produkte, die in diesem Zusammenhang hergestellt wurden.

Grundlage für den Erfolg eines Stars war laut Morin die Fähigkeit, Identifikationen und Projektionen in dessen Person zu bündeln. Ein Star verkörpert den Wunsch der Zuschauer, ein besseres Leben jenseits des Alltags leben zu können, womöglich auch, indem er gegen gesellschaftliche Regeln verstößt. Soweit ein solcher Ausbruch für den Rezipienten nicht möglich ist, wird vom Star Lebenshilfe, Unterstützung und Ermutigung erwartet. Von Stars wurde - zumindest in der Frühphase des Star-Systems - erwartet, dass sie Lebensfreude und freundschaftliche Zuwendung (zum Publikum und untereinander) ausstrahlen.

Die verbreiteten Erfolgsgeschichten, in denen einfache Menschen von der Straße zu Ruhm und Ehre gelangten, weckten im Zuschauer die Frage: »Warum sollte nicht auch ich dieses große Los ziehen können? « Andererseits war dem Rezipienten durchaus bewusst, dass dieses System ein geschlossenes war, dessen Regeln eher vom Zufall abhingen, statt durchschaubar zu sein, und dass bereits die Gesetze der Wahrscheinlichkeit ihn von diesem Glück trennten. Aspiranten auf den Schauspielerberuf wurden zudem mahnend darauf hingewiesen (Morin nennt Beispiele von Briefen Luis Marianos an Fans), dass dies kein leichter Beruf sei und neben Talent auch eine solide Ausbildung, unbedingter Wille und Fleiß für eine Karriere erforderlich seien (vgl. Morin 1972: 78ff.).

Das alte Star-System Hollywoods wurde laut Morin (1972: 148ff.) dadurch abgelöst, dass die ökonomische Kraft des Kinos (nicht zuletzt durch die Konkurrenz des Fernsehens) verfiel und sich die Regisseure eher der Darstellung der Alltagsrealität statt dem Versprechen eines besseren Lebens zuwandten. Die Protestbewegungen der 1960er Jahre forcierten diese Entwicklung und führten zu einem Nebeneinander des Kinos alter und neuer Schule. Entsprechend verloren Stars ihren Vorbildcharakter, und auch die Forderung nach einem Happy End verlor an Bedeutung. Stars sind demnach bereits seit den 1970er Jahren keine glücklichen Halbgötter mehr, sondern Symbole für die Leiden der Normalsterblichen: 
»Wie zuvor ernährt man sich von ihrem Leben; aber man kostet nicht mehr vom Elixier der Glücksverheißung noch von dem schönen Leben, das durch ihre Person vermittelt wird; man ernährt sich von ihren Dramen, ihrem Elend und [...] rächt sich gar an ihrer Größe, indem man nach Selbstmord und Tragik verlangt. «3

Der Abstand zwischen den Stars und denen, die sie - nach wie vor - verehren, wird geringer, die Identifikationsmöglichkeiten größer. Damit verschmelzen Filmstars im »neuen Olymp der Massenkultur« (Morin 1972: 155) und reihen sich in die Prominenz von Adel und Königshäusern oder auch Musikern ein.

\section{Das Star-System der Casting-Shows am Beispiel DSDS}

Morin geht davon aus, dass mit Aufkommen des modernen Kinos zwar die Elemente des Star-Systems weiter bestanden, eine Institutionalisierung jedoch nicht mehr statt fand. Das Kino prägte die Massenkultur nicht mehr, sondern reflektierte lediglich gesellschaftliche Strömungen. Im popmusikalischen Bereich lässt sich jedoch bereits in der Boygroup-Ära der 1990er Jahre eine Art Revival des Star-Systems ausmachen: Es wurde eine systematische Suche nach jungen, gut aussehenden und sich gut bewegenden Talenten betrieben und mittels »Casting-Fahrplan« (Weyrauch 1997: 41) ausgebaut. Dieses System wurde in späteren Casting-Formaten (DSDS, Popstars, Starsearch, Die deutsche Stimme) fortgeführt und um das Prinzip der Partizipation erweitert: Die Zuschauer sollten für den/die von ihnen favorisierten Kandidaten/Kandidatin anrufen; wer die wenigsten Stimmen erhielt, schied aus dem Wettbewerb aus. Den Zuschauern wurde hierdurch zweierlei suggeriert: zum Einen, dass jeder es doch mal versuchen könne, Star zu werden; zum Anderen, dass das Publikum mit entscheiden könne, wer es verdient habe, ein Star zu werden.

An der Systematik der Casting-Shows änderte dies jedoch nichts: Die Vorauswahl wurde durch Experten (einer Jury) abgesichert, die die Darbietungen der Kandidaten kontinuierlich kommentierten. Kandidaten, die in der engeren Auswahl (bei DSDS: unter den letzten zehn) standen, lebten bis zu ihrem jeweiligen Ausscheiden in einer Art Wohngemeinschaft und wurden

3 »Comme auparavant, l'on se nourrit de leurs vies; mais on ne goûte plus à l'élixir prometteur de félicité ni à la belle vie par leur personne interposée; on se nourrit de leurs drames, de leur misère et [...] se venge même de leur grandeur en redemandant du suicide et du tragique« (Morin 1972: 156; Übersetzung I.S.). 
rundum betreut: Kleidung, Stimmbildung, Tanztraining, Studioaufnahmen, Interviews und Termine, Fanartikel - alles wurde umfassend von weiteren Experten organisiert, alle Stunden des Tages durchstrukturiert.

Die Bewertungsmaßstäbe der Jury orientierten sich zunächst an den Konventionen der Boygroup-Ära: musikalische und klangliche Qualität des Gesangs (das Spielen eines Instruments war in diesem Setting nicht vorgesehen bzw. nicht erwünscht) sowie Qualität der Bühnenpräsenz (Tanz und Performance) und Aussehen. Da es in Formaten wie DSDS jedoch um einzelne Stars ging und nicht um das Formieren einer Band, wurde verstärkt die Bedeutung von »personality«, Originalität und Besonderheit, hervorgehoben, die - wie im Falle Daniel Küblböcks - sogar in der Lage war, gesangliche Defizite (bzw. das, was die Jury als solche wertete) zu überdecken.

Als weiteres entscheidendes Kriterium galt die Fähigkeit der Kandidaten, Identifikation und Emotionalisierung zu ermöglichen (»Drei Dinge sind wichtig: Gefühl, Gefühl, Gefühl«, Dieter Bohlen in Daniel Küblböck - Das Superstar-Special). Dies war der wohl wichtigste Faktor für den Erfolg Daniel Küblböcks im Rahmen der Show: Er brachte Emotionen meist unmittelbar und sehr direkt zum Ausdruck. Allerdings hatten die gezeigten Gefühle sich im telegenen Rahmen zu bewegen und kontrollierbar zu bleiben: Verstohlen weggewischte Tränen in Großaufnahme und erstickte Stimmen waren integraler Bestandteil der Superstar-Inszenierung. Der Zusammenbruch Küblböcks beim Ausscheiden seiner Lieblingskandidatin Gracia wurde jedoch aus allen Rückblenden herausgeschnitten.

\section{Publikumsliebling und Publikumsschreck - zur Rezeption Daniel Küblböcks}

Daniel Küblböck begann seine DSDS-Karriere mit einem Regelverstoß: $\mathrm{Er}$ überrumpelte die Jury, als er bei seiner ersten Vorstellung singend und Gitarre spielend in den Casting-Raum trat, obwohl instrumentale Begleitung vom Regelwerk untersagt war. Seine Art, sich zu präsentieren, ließ Bohlen anerkennend feststellen: »Du hast ganz ehrlich 'ne Schraube locker, [...] das gehört bei diesem Geschäft auch dazu. Ich find dich lustig, von mir kriegst du ein Ja« (Dieter Bohlen in Daniel Küblböck - Das Superstar-Special). Somit war es Küblböck gelungen, die Experten für sich einzunehmen, die trotz mahnender Worte bezüglich des Gesangs immer wieder seine Entertainment-Fähigkeiten lobten.

Der selbstbewusste (»Jury, ich bin's«) und schlagfertige Umgang mit den Experten (»Der Wald wäre sehr still, wenn nur die begabtesten Vögel sin- 
gen ) machte jedoch auch einen nicht unwesentlichen Teil seiner Beliebtheit beim Publikum aus. Er fiel auf, fiel aus dem Superstar-Rahmen: Der Kommentator ${ }^{4}$ des Superstar-Specials von SuperRTL bezeichnet inn als "schräg, schrill und dezent rebellisch« und blendet einen Interview-Ausschnitt ein, in dem Daniel Küblböck über sich selbst sagt:

»Bei mir ist es einfach so, dass ich irgendwie schon ein bisschen gegen die Gesellschaft demonstrier - ein bisschen, ein kleines bisschen, so gegen das, was ich nicht will. Das kann man schon ein bisschen mit Pippi Langstrumpf vergleichen. Ich glaub einfach, dass das bei mir daran liegt, dass ich die buntesten Klamotten anhab, und für einen Mann sowieso, und ich auch eine feminine Seite zeige, die einfach dazugehört zum Leben.«

Dass Daniel Küblböck die Nation gespalten habe, wurde zur stehenden Redewendung. Das Publikum teilte sich in solche, die begeistert jubelten und solche, die seine Auftritte mit Buh-Rufen kommentierten. Per Telefonvoting kam er jedoch Samstag für Samstag weiter, bis ins Halbfinale. Seine Anhänger freuten sich über das Überraschungsmoment, das er ihnen bot:

»Wenn Daniel auftritt, dann ist immer alles total kribbelig und was wird er jetzt machen und total spannend und meistens auch sehr lustig. Man freut sich richtig auf den Daniel; bei den anderen weiß man, ja, die machen ihre Show, singen gut und alles« (Marius Theobald in Daniel Küblböck - Das Superstar-Special).

Er galt als Garant dafür, dass in der Show nicht nur die weiterkamen, die den bisherigen Boygroup-Idealen entsprachen. Selbst Daniel Lopez, ein weiterer Kandidat der ersten Superstar-Staffel, der den lässigen Latino-Typ mit Samtstimme verkörperte, schied früher aus dem Wettbewerb. Entsprechend bewertete eine Teilnehmerin eines Internetforums die Rolle Küblböcks folgendermaßen: »Daniel wird wohl populärer werden, trotz des 3. Platzes! Daniel hat die Fähigkeiten eines Superstars! Er spielt mit den Medien! [...] Alex wird schnell vergessen sein! ${ }^{5}$

Die Möglichkeit einer Identifikation ist für Morin essentieller Bestandteil des Star-Systems (vgl. Morin 1972: 48f.). Daniel Küblböck, der nicht perfekt sang, nicht von makelloser Schönheit war, nett blieb und viele Schwierigkeiten - nicht nur im DSDS-Kontext, sondern schon in seiner Kindheit - zu bewältigen hatte, bot ein breites Angebot an Identifikationsmöglichkeiten und verkörperte zugleich das Versprechen, dass sich Grenzen außer Kraft

4 Stimme aus dem Off, die einzelne Filmsequenzen kommentiert, im Abspann nicht namentlich genannt.

5 http://www.stern.de/community/forum/thread.jsp?forum=93\&thread=19780\& message=238946, Eintrag vom 2. März 2003 (Zugriff: 22.1.2005). 
setzen lassen: »Und wer mein Buch zu Ende gelesen hat, traut sich vielleicht auch aufzustehen. Von nun an dem eigenen Bauch zu trauen. Und irgendwas anzufangen, das alle überrascht« (Küblböck 2003: 10).

Daniel Küblböck - Das Superstar-Special zeigt zahlreiche Ausschnitte, in denen Zuschauer äußerten, er sei lustig. Der Kommentator bescheinigte ihm, er sei »mit der unterhaltsamste Kandidat von Deutschland sucht den Superstar «. Thomas Stein verlieh inm das Attribut »singing comedian«. Beliebt waren auch die kreativen Coverversionen, in denen er die von ihm (aus einer vorgegebenen Auswahl) gewählten Songs umdeutete. Die von Dieter Bohlen produzierten CDs, die im Anschluss an die erste DSDS-Staffel veröffentlicht wurden, bereiteten diesen kreativen Experimenten allerdings ein Ende; Daniel Küblböck wurde - in Anlehnung an die Retro-Welle - auf den musikalischen Stil der 1960er Jahre-Popsongs festgeschrieben, eigene Songs wurden bislang nicht veröffentlicht.

Die Gegner Küblböcks unter den DSDS-Rezipienten äußerten in erster Linie - neben persönlicher Antipathie - Kritik an seiner Stimme und an seinem Gesang:

»Am Anfang war er ja noch irgendwie interessant, weil nicht massenkompatibel. Dumm nur, daß sich dann bei einigen DSDS-Shows rausstellte, daß er massive Schwierigkeiten hat, den Ton zu treffen.«

$»[\ldots]$ der hat null Gesangstalent. ${ }^{6}$

Andere wollten ihn nicht auf einer Stufe mit bereits in den Charts befindlichen Stars genannt wissen:

»ich bin zwar kein Danielhasser oder Liebhaber, mir ist das wurscht, was der macht und so, aber wirklich mit Justin und Robbie eingestuft zu werden is auf jeden Fall heftig, denn die haben wesentlich mehr geleistet als Daniel K. « ${ }^{7}$

Die Debatten, die Küblböck im Publikum auslöste, waren für den produzierenden Sender ein Glücksfall, sicherten sie doch dem DSDS-Format ein Maß an Aufmerksamkeit, das keiner anderen Casting-Show zu Teil wurde. Der »schräge Daniel « (Aarberger 2003: 37) wurde im Anschluss an die DSDSStaffel überall herumgereicht: Er trat in Talkshows, Quizsendungen, Moderationen auf und übernahm die Hauptrolle in einem Film, so dass selbst Aarberger (2003: 100ff.) von einem Übersättigungseffekt spricht. Auch Ich

6 http://forum.freenet.de/app/m/_t126876c274pf-1unterhaltung_Wasmoegt_ ihr_an_Daniel_Ku, Einträge vom 5. Dezember 2004, 11:21, und 23. Dezember 2004, 14:06 (Zugriff: 26.4.2005).

7 http://www.chat-radio.de/hp3/modules.php?name=Forums\&file=viewtopic\&t=

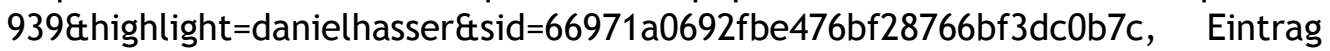
vom 17. Januar 2004, 23:39 (Zugriff: 26.4.2005). 
bin ein Star, holt mich hier raus ließ er nicht aus, ein Sendeformat, in dem »Halbprominente« (Tuma 2004: 156) Mutproben im Dschungel zu bestehen hatten, die nicht etwa einem Survival-Training in freier Natur entsprachen, sondern durch verschärfte Versuchsanordnungen (wie z.B. einem Kakerlakenbad) sadistisch verstärkt wurden. Einige prominente Teilnehmer dieses Dschungelcamps fanden in diesem Sendeformat eine Aufwertung in der Publikumsgunst (etwa der zum »Dschungelkönig« gekürte Costa Cordalis); für Daniel Küblböck geriet dieses Experiment eher zur Demontage eines Stars, an dessen illegitimer Prominenz sich das Publikum rächen wollte.

Eben jene Legitimität ist auch zentraler Gegenstand der Diskussionen, die jenseits des DSDS-Kontextes geführt wurden bzw. in denen er Erwähnung fand. Daniel Küblböck - und nicht etwa der Produzent Bohlen oder der auf Popsendungen spezialisierte Sender RTL - wurde zur Chiffre für allerlei Unbehagen, das vom DSDS-Business ausgelöst wurde. So zielt die eingangs zitierte Äußerung der thüringischen Wissenschaftsministerin auf eine generelle Kritik am Wettbewerbsprinzip als Grundlage für Qualitätssicherung. Die Rede Bastians hat das Fehlen eigener musikalischer Aktivität bei TVKonsumenten im Blick und beinhaltet in erster Linie eine Aufforderung zu besserer musikpädagogischer Förderung in der Schule. Wenn im Artikel über Musikhochschulen die »harte Arbeit« der Musikstudenten dem »Traum von Ruhm und großem Geld « der Superstar-Kandidaten gegenüber gestellt wird, stellt dies die musikalische Qualität des Sendeformats Casting-Show in Frage. Die Kritik des Psychologen und Traumaforschers Lüdke schließlich, das Verhalten von Stars könnenegative Vorbildfunktion für die Fans haben, entspringt der alten Befürchtung, die auch bei Bastian anklingt: jugendliche Medienkonsumenten würden schädlichen Einflüssen ausgesetzt, vor denen man sie schützen müsse.

Es ist anzunehmen, dass viele der am öffentlichen Diskurs Beteiligten weder DSDS gesehen noch Daniel Küblböcks Gesang gehört haben: In ihrer empirischen Untersuchung stellt Sarah Wolf (2004: 58ff.) fest, dass selbst aus ihrem Sample von 200 befragten Jugendlichen 15,5\% DSDS »kannten«, ohne auch nur eine der Shows gesehen zu haben. Für die Botschaften, die vermittelt werden sollen, stellt dies jedoch kein Hindernis dar, entsprechend der Feststellung Schweppenhäusers (2004: 41) in Anknüpfung an Günther Anders: "Nicht der Gegenstand selbst, sondern etwas von ihm und über ihn wird anwesend gemacht. Auf diese Weise wird ein neuer Gegenstand gemacht.«

Die Medien prägen ein kollektives Gedächtnis, auf das sich alle beziehen (ebd.: 35); alle Themen finden dort ihren Anknüpfungspunkt. Auch Kritik an den Medien ist letztlich nur durch die Medien vermittelbar (ebd.: 111). Ent- 
sprechend lässt sich nachvollziehen, dass DSDS zum Fundus werden konnte für kritische Botschaften in Bezug auf politische, pädagogische und musikalische Phänomene, die sich - quasi stellvertretend - an Daniel Küblböck festmachen. Die Spaltung des DSDS-Publikums wurde so jenseits des TVFormats gedoppelt, was als Nebeneffekt dazu beitrug, Daniel Küblböck und mit ihm DSDS im Gespräch zu halten.

An dieser Stelle lässt sich noch ein weiteres, von Morin (1972: 167ff.) in einem Anhang zu seiner Analyse des Star-Systems dargelegtes Motiv ausmachen: das Motiv des rituellen Sündenbocks. Dies beschränkt sich jedoch nicht allein darauf, dass Küblböck sozusagen stellvertretend für ein kommerzialisiertes und autoritäres Casting-System im Zentrum öffentlicher Kritik steht. Morin veranschaulicht die Figur des Sündenbocks am Beispiel des Antihelden in Slapstick-Komödien: Dieser darf sich ungeschickt anstellen, gegen gesellschaftliche Regeln und den guten Geschmack verstoßen, kindliche Unschuld an den Tag legen, auf die Insignien dominierender Männlichkeit verzichten, weil das Publikum ihn mit Schadenfreude dafür opfert, dass er es von seinen Schwächen entlastet. Womöglich ist das die Hauptrolle des »lustigen« Daniel Küblböck: nicht allein Entertainer zu sein, sondern die Unzulänglichkeiten aller Nicht-Stars und Antihelden zu verkörpern und hierfür (analog dem Schadenfreude-Prinzip, das jeder Karaoke-Veranstaltung zu Grunde liegt) quasi stellvertretend für alle ausgebuht zu werden, die im Wettbewerb nicht mithalten können.

\section{Schlussbemerkungen}

Morin sah in den sich wandelnden Ausprägungen des Star-Systems Spiegelungen der jeweils aktuellen Situation der bürgerlichen Gesellschaft. In DSDS und den Debatten, die es ausgelöst hat, wurde vielleicht auch deutlich, was die Themen der (bundesdeutschen) Gesellschaft zu Beginn des 3. Jahrtausends sind: ungebrochenes Diktat der Ökonomie, Propagierung der Eigeninitiative und des Glücks des Tüchtigen, positives Denken, Sehnsucht nach altem Glamour (analog zur Retro-Welle der Charts), an den man letztlich doch nicht glaubt.

Über die musiksoziologischen Fragestellungen hinaus bietet sich der Musikwissenschaft die Chance, empirisch zu klären, warum gerade der Gesang in den Fokus des Casting-Systems gelangt ist: Lag dem Erfolg von DSDS auch ein gestiegenes Rezipienten-Interesse am Singen zu Grunde, und wenn ja: Ist dieses Interesse eher bestärkt worden, oder hat die Wucht des durchorganisierten Star-Systems Eigeninitiative eher entmutigt? 


\title{
Literatur
}

Aarberger, Ariane (2003). Daniel Küblböck. Erfolge, Fans, Gegner. Steyr: Ennsthaler Verlag.

Bastian, Hans Günther (2004). [Rede anlässlich der Verleihung des Frankfurter Binding-Kulturpreises, auszugsweise dokumentiert in der Rubrik »Im Wortlaut: Musik gehört zum Leben. «] In: Frankfurter Rundschau vom 14. Juni, S. 29.

Horkheimer, Max/Adorno, Theodor W. (1987). "Kulturindustrie. Aufklärung als Massenbetrug.«In: >Dialektik der Aufklärung` und Schriften 1940-1950 (= Max Horkheimer, Gesammelte Schriften, Band 5). Frankfurt/M.: Suhrkamp, S. 144196 (E: 1947).

Küblböck, Daniel [in Zusammenarbeit mit Julia Boenisch] (2003). Ich lebe meine Töne. München: Limes Verlag.

Morin, Edgar (1972). Les stars. Paris: Editions du Seuil (E: 1957).

N.N. (2004a). »Elite-Unis: Castingshow mit Edelgard."In: Spiegel-Online vom 26. Januar, http://www.spiegel.de/unispiegel/studium/0,1518,283607,00.html (auch zit. in: http://www.manager-magazin.de/koepfe/artikel/0,2828,2837592,00.html) (Zugriff: 26.4.2005).

N.N. (2004b). »Küblböck ist auf dem Stand eines 13jährigen. «In: Bild.T-Online vom 3. März, http://www.bild.t-online.de/BTO/kinomusik/aktuell/2004/03/03/big brother/big_brother.html (auch zit. in: http://www.beepworld.de/cgi-bin/ forum_de/archive/t-63328.html) (Zugriff: 26.4.2005).

Schweppenhäuser, Gerhard (2004). »Naddel« gegen ihre Liebhaber verteidigt. Ästhetik und Kommunikation in der Massenkultur. Bielefeld: transcript Verlag.

Schreiber, Anja (o.J.). "Keine Chance für Daniel Küblböck.« In: http:// www.jobpilot.de/gateway/partner/coop.phtml/journal/studium/thema/pop4103.html?PARTNER=content; Version vom 6. Oktober 2003 (Zugriff: 22.1.2005).

Tuma, Thomas (2004): »Die Buschtrommler.«In: Der Spiegel, H. 4, S. 156-158. Weyrauch, Jan (1997). Boygroups. Das Teenie-FANomen der 90er. Berlin: Extent. Wiediger, Martin (1996). Die Fan-Star-Beziehung in der Rockmusik. Eine empirischsoziologische Vergleichsstudie von Rockfans in Italien und Deutschland. Frankfurt/M.: Unveröffentl. Diplomarbeit am Fachbereich Gesellschaftswissenschaften der Johann-Wolfgang-Goethe-Universität.

Wolf, Sarah (2004). Deutschland sucht den Superstar - Analyse der Erfolgsfaktoren. Hamburg: Diplomica.

\begin{abstract}
The first series of Deutschland sucht den Superstar, the German version of Simon Fuller's casting show Pop Idol, has brought up a character that attracted more attention than the winner of the show. Daniel Küblböck, a Bavarian teenager of 17, divided the public into fans and adversaries. Politicians, educators and musicians especially felt challenged by the sudden celebrity of a youngster that did not correspond to standards of quality and perfection. The essay starts from a general theory of the star phenomenon as developed by the French sociologist Edgar Morin
\end{abstract}


who has described the star system as the result of an anthropological need of myth creation and adoration, persisting even in so-called rationalist societies, realized by a capitalist economy. It is shown that there are correspondences between the star systems of the cinema and of the recent casting shows. Within this context, Daniel Küblböck was not just contested for his own qualities or failures: He plays the part of a ritual scapegoat, being charged for all reluctance towards the casting system as a whole and, on the other hand, attracting the desire for punishment of those who are not winners of the competitive society. 